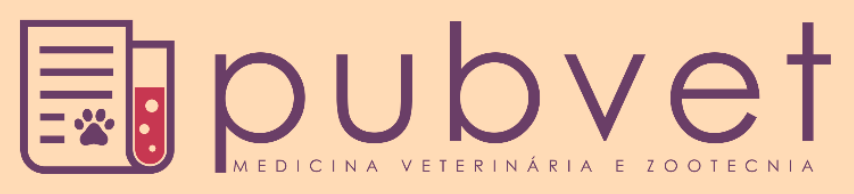

https://doi.org/10.31533/pubvet.v12n11a204.1-5

\title{
Comparação da eficácia de difloxacina e orbifloxacina no tratamento de infeções de $E$. Coli $e$ S. Intermedius em cães
}

\author{
Gabrielda Silva Paiva ${ }^{1}$, Gabriele Mortean da Silva ${ }^{1} \bullet$, Maria Eduarda Canassa ${ }^{1} \bullet$, Sheila \\ Rezler Wosiacki $^{2} \bullet$, Marcos Ferrante ${ }^{2 *} \bullet$
}

${ }^{I}$ Academico do curso de Medicina Veterinária da Universidade Estadual de Maringá, Umuarama, PR Brasil.

${ }^{2}$ Docente do curso de Medicina Veterinária da Universidade Estadual de Maringá, Umuarama, PR Brasil.

* Autor para correspondência: marcosferrante@gmail.com

RESUMO. O objetivo do presente trabalho foi avaliar e comparar o efeito de mudanças na CIM, nas taxas de eficácia de difloxacina e orbifloxacina no tratamento das infecções bacterianas causadas por Escherichia coli (E. coli) ou Staphylococcus intermedius (S. intermedius) em cães. Realizou-se o modelamento farmacocinético/farmacodinâmico (PK/PD) para a determinação da eficácia do tratamento com $5 \mathrm{mg} / \mathrm{kg}$ de diclofaxina ou $2,5 \mathrm{mg} / \mathrm{kg}$ de orbifloxacina via oral a cada 24 horas de infeções causadas por $E$. coli $S$. intermedius em cães. Foi realizada uma simulação de Monte Carlo dos parâmetros farmacocinéticos de 10.000 eventos. Os valores de $\mathrm{PK}$ foram obtidos de estudos realizados em cães, onde determinaram valores de $\mathrm{ASC}_{24}$ de $9.34 \pm 2.09 \mathrm{ug} / \mathrm{ml}$ para difloxacina e de $\mathrm{ASC}_{24}$ de $12.72 \pm 2.29 \mathrm{ug} \mathrm{h} / \mathrm{ml}$ para orbifloxacina. Seguidamente foi realizado modelamento PK/PD para determinar as taxas de probabilidade de atingir os índices $\mathrm{ASC}_{24} / \mathrm{CIM}_{90}$ de correspondentes, no intervalo de CIM de $0.062-0.5 \mathrm{ug} / \mathrm{ml}$. A probabilidade de obter a cura bacteriologia após o tratamento com qualquer dos fármacos diminuiu significativamente para infeções causadas por E. coli ou S. intermedius com CIM superiores a $0.125 \mathrm{ug} / \mathrm{ml}$. As análises comparativas das taxas de eficácia estimadas para os dois fármacos avaliados determinaram que não existem diferencias significativas para o tratamento de infeções causadas por E. coli ou $S$. intermedius no intervalo de CIM estudados. Estes resultados indicam que apesar da diferencia nas doses administradas a eficácia de ambos tratamentos não apresenta diferencias significativas. Porem para casos com CIM superiores deveram ser realizados ajustes nas doses.

Palavras chave: farmacodinâmica, farmacocinética, flourquinolonas, otimização de doses

\section{Comparison of the efficacy of difloxacin and orbifloxacin in the treatment of E. coli and $S$. intermedius infections in dogs}

\begin{abstract}
The objective of the present study is to evaluate and compare the effect of changes in MIC, efficacy rates of difloxacin and orbifloxacin in the treatment of bacterial infections caused by $E$. coli or S. intermedius in dogs. The PK / PD modeling was performed to determine the efficacy of oral orbifloxacin $5 \mathrm{mg} / \mathrm{kg}$ diclofaxine or $2.5 \mathrm{mg}$ / $\mathrm{kg}$ orally every 24 hours from $E$. coli or $S$. intermedius infections in dogs. A Monte Carlo simulation of the pharmacokinetic parameters of 10,000 events was performed. PK values were obtained from studies in dogs, where they determined $\mathrm{AUC}_{24}$ values of $9.34 \pm 2.09$ $\mu \mathrm{g} / \mathrm{ml}$ for difloxacin and $\mathrm{AUC}_{24}$ of $12.72 \pm 2.29 \mu \mathrm{g} / \mathrm{ml}$ for orbifloxacin. Then, PK / PD modeling was performed to determine the likelihood of reaching corresponding $\mathrm{AUC}_{24} /$ $\mathrm{CIM}_{90}$ indices in the MIC range of $0.062-0.5 \mathrm{ug} / \mathrm{ml}$. The probability of obtaining bacteriology cure after treatment with either drug decreased significantly for infections caused by E. coli or S. intermedius with MICs greater than $0.125 æ g / \mathrm{ml}$. The comparative
\end{abstract}


analysis of the estimated efficacy rates for the two drugs evaluated determined that there are no significant differences for the treatment of infections caused by $E$. coli or $S$. intermedius in the MIC range studied. These results indicate that despite the difference in the doses administered the efficacy of both treatments did not present significant differences. However, in cases with higher MICs, dose adjustments should be made.

Keywords: pharmacodynamics, pharmacokinetics, flourquinolones, dose optimization

\title{
Comparación de la eficacia de difloxacina y orbifloxacino en el tratamiento de las infecciones de $E$. Coli y $S$. Intermedius en perros
}

\begin{abstract}
RESUMEN. El objetivo del presente trabajo fue evaluar y comparar el efecto de cambios en la CIM, en las tasas de eficacia de difloxacina y orbifloxacino en el tratamiento de las infecciones bacterianas causadas por $E$. coli o $S$. intermedius en perros. Se realizó el modelado PK / PD para la determinación de la eficacia del tratamiento con $5 \mathrm{mg} / \mathrm{kg}$ de diclofaxina o $2,5 \mathrm{mg} / \mathrm{kg}$ de orbifloxacina por vía oral cada 24 horas de infecciones causadas por E. coli o $S$. intermedius en perros. Se realizó una simulación de Monte Carlo de los parámetros farmacocinéticos de 10.000 eventos. Los valores de PK se obtuvieron de estudios realizados en perros, donde determinaron valores de $\mathrm{AUC}_{24} \mathrm{de} 9.34 \pm 2.09 \mathrm{ug} / \mathrm{ml}$ para difloxacina y de $\mathrm{AUC}_{24}$ de $12.72 \pm 2.29 \mathrm{ug} \mathrm{h} / \mathrm{ml}$ para orbifloxacina. A continuación, se realizó el modelado PK / PD para determinar las tasas de probabilidad de alcanzar los índices $\mathrm{AUC}_{24} / \mathrm{CIM}_{90}$ de corresponsales, en el intervalo de CIM de $0.062-0.5 \mathrm{ug} / \mathrm{ml}$. La probabilidad de obtener la cura bacteriológica después del tratamiento con cualquiera de los fármacos disminuyó significativamente para infecciones causadas por E. coli o $S$. intermedius con CIM superiores a $0.125 \mathrm{ug} / \mathrm{ml}$. El análisis comparativo de las tasas de eficacia estimadas para los dos fármacos evaluados determinó que no existen diferencias significativas en el tratamiento de las infecciones causadas por $E$. coli o $S$. intermedius en el intervalo de CIM estudiados. Estos resultados indican que a pesar de la diferencia en la dosis administrada la eficacia de ambos tratamientos no presenta diferencias significativas. Sin embargo, para casos con CIM superiores se deben realizar ajustes en las dosis.
\end{abstract}

Palabras claves: farmacodinámica, farmacocinética, flourquinolonas, optimización de dosis

\section{Introdução}

Fluoroquinolonas são agentes antibacterianos sintéticos introduzidos em medicina veterinária, primeiro como enrofloxacina (Evans \& Chapple, 2002; Heinen, 1999). As fluorquinolonas atualmente são uma das maiores classes de agentes antimicrobianos sendo utilizadas, mundialmente, no tratamento de infecções de origem bacteriana (Martinez et al., 2006; Trouchon \& Lefebvre, 2016). Este grupo de fármacos foi se desenvolvendo de forma a ultrapassar grande parte das suas adversidades, possuindo um espectro de ação amplo, boa disponibilidade oral, melhor difusão para os tecidos, uma meia vida maior e uma toxicidade consideravelmente reduzida (Silva \& Hollenbach, 2010; Trouchon \& Lefebvre, 2016). De acordo com Scholar (2002) as fluoroquinolonas atuam por inibição da atividade catalítica de duas enzimas responsáveis e essenciais à replicação e transcrição do DNA bacteriano: a DNA girase e a topoisomerase IV (topoisomerases do tipo II). A enrofloxacina tem uma substituição de flúor, a difloxacina tem duas substituições de flúor e a orbifloxacina tem uma substituição de três moléculas de flúor, mas a presença de mais de um flúor não aumenta os efeitos antibacterianos ( \& Hollenbach, 2010; Trouchon \& Lefebvre, 2016). As fluoroquinolonas são considerados antibióticos lipossolúveis, porem dentre deste grupo os menos lipossolúveis como marbofloxacina, orbifloxacina têm um volume de distribuição (Vd) menor do que aquelas com maior lipossolubilidade como enrofloxacina e difloxacina (Frazier et al., 2000). Uma explicação para esta observação é que os fármacos mais lipossolúveis apresentam maiores concentrações intracelulares, mas maior ligação tecidual também poderia explicar as diferenças no volume de distribuição (Trouchon \& Lefebvre, 2016). Existem estudos que determinam os parâmetros farmacocinético de difloxacina e orbifloxacina via oral em cães (Heinen, 2002). Todavia, não existem estudos que determinem a eficácia frente a mudanças da susceptibilidade bacteriana. A 
utilização dos modelos PK/PD conjuntamente com o método de simulação de Monte Carlo foi descrita por Dudley \& Ambrose (2000) e por Trang et al. (2017) e atualmente é a principal metodologia empregada para a avaliação e otimização de doses terapêuticas de antibióticos (Abdelraouf et al., 2017; Gieschke \& Serafin, 2014; Toutain et al., 2007; Toutain et al., 2016; Toutain et al., 2017).

Este trabalho foi realizado para avaliar e comparar o efeito de mudanças na CIM, nas taxas de eficácia de difloxacina e orbifloxacina no tratamento das infecções bacterianas causadas por E. coli ou S. intermedius em cães.

\section{Material e métodos}

Realizou-se um modelamento PK/PD para a determinação da eficácia do tratamento com 5 $\mathrm{mg} / \mathrm{kg}$ de diclofaxina ou $2,5 \mathrm{mg} / \mathrm{kg}$ de orbifloxacina via oral a cada 24 horas de infeções causadas por E. Coli S. intermedius em cães. O parâmetro de eficácia utilizado foi o índice da área sob da curva da concentração plasmática em função do tempo/concentração inibitória mínima(ASC $24 / \mathrm{MIC}_{90}$ ) (Ahmad et al., 2016; Toutain et al., 2007). Os índices a AUC24/CIM90 de utilizados foram 73 para E. coli e 37 para $S$. intermedius para diflozxacina e 102 para E. coli e 51 para $S$. intermedius para Orbifloxacina (Heinen, 2002). Foi realizada uma simulação de Monte Carlo dos parâmetros farmacocinéticos de 10.000 eventos. Os valores de PK foram obtidos de estudos realizados em cães, onde determinaram valores de $\mathrm{AUC}_{24}$ de $9.34 \pm 2.09 \mathrm{ug} / \mathrm{ml}$ para difloxacina e de $\mathrm{AUC}_{24}$ de $12.72 \pm 2.29 \mathrm{ug} \mathrm{h} / \mathrm{ml}$ para orbifloxacina (Figura 1) (Heinen, 2002). Seguidamente foi realizado modelamento PK/PD para determinar as taxas de probabilidade de atingir os índices $\mathrm{AUC}_{24} / \mathrm{CIM}_{90} \mathrm{de}$ correspondientes, no intervalo de CIM de 0.062 $0.5 \mathrm{ug} / \mathrm{ml}$ segundo a metodologia descrita por Dudley \& Ambrose (2000) e Trang et al. (2017). A análise estatística foi realizada no programa Excel e no programa BioEstat 5.0. Foram comparadas as taxas de eficácia correspondentes a cada CIM estudada mediante o teste de independência do Qui-Quadrado com uma significância de $(\mathrm{P}=0,01)$.

\section{Resultados e discussão}

$\mathrm{Na}$ figura 1 se apresenta a razão da concentração plasmática de difloxacina e orbifloxacina em cães e as CIM avaliadas. O presente estudo possibilitou determinar mudanças nas taxas de eficácia relacionadas com mudanças na CIM para difloxacina e para orbifloxacina.

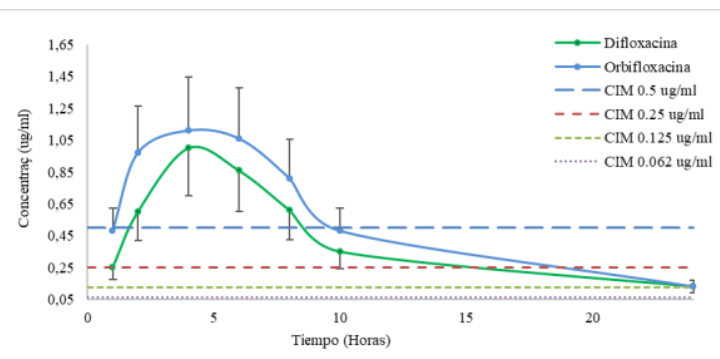

Figura 1: Relação da farmacocinética (media e desvio padrão) de difloxacina na dose de $5 \mathrm{mg} / \mathrm{kg}$ e de orbifloxacina na dose de $2,5 \mathrm{mg} / \mathrm{kg}$ via oral em cães e as concentrações inibitórias mínimas das bactérias infectantes (CIM) (adaptado de $\underline{\text { Heinen (2002). }}$.

Para difloxacian as taxas eficácia estimadas para E. coli foram de 99\%; 54\%; $0 \%$; e $0 \%$ e segundo as CIM das cepas 0,$062 ; 0,125 ; 0,25$ e 0,5 respectivamente. A probabilidade de obter a cura bacteriologia após o tratamento diminuiu significativamente para infeções causadas por bactérias com CIM superiores a $0,062 \mathrm{ug} / \mathrm{ml}(\mathrm{P}<$ $0,001)$. Enquanto que as taxas eficácia estimadas para S. intermedius foram de 100\%; 99\%; 50\%; e $0 \%$ e segundo as CIM das cepas 0,$062 ; 0,125 ; 0,25$ e 0,5 respectivamente. A probabilidade de obter a cura bacteriologia após o tratamento diminuiu significativamente para infeções causadas por bactérias com CIM superiores a $0.125 \mathrm{ug} / \mathrm{ml}(\mathrm{P}<$ 0,001) (Figura 2).

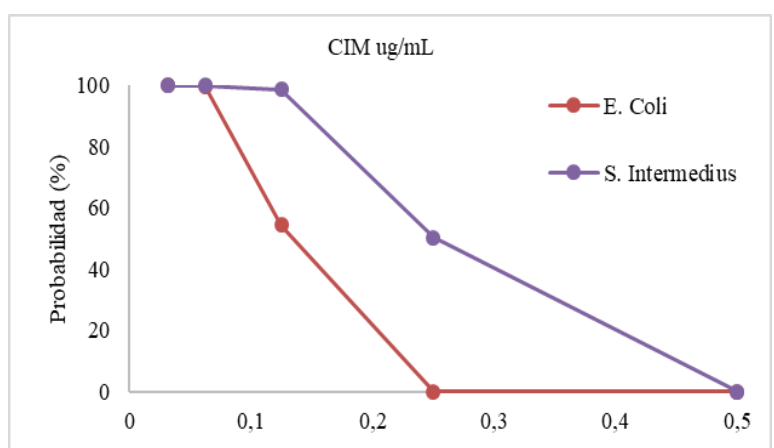

Figura 2. Probabilidade de atingir o índice $\mathrm{ASC}_{24} / \mathrm{CIM}_{90}$ após o tratamento com $5 \mathrm{mg} / \mathrm{kg}$ de Difloxacina via oral em cães segundo a CIM do microrganismo infectante baseado na simulação de Monte Carlo $(\mathrm{n}=10.000)$.

Nos analises de realizados com orbifloxacina as taxas eficácia estimadas para $E$. coli foram de 99\%; $50 \%$; $0 \%$; e $0 \%$ e segundo as CIM das cepas 0,$062 ; 0,125 ; 0,25$ e 0,5 respectivamente. A probabilidade de obter a cura bacteriologia após o tratamento diminuiu significativamente para infeções causadas por bactérias com CIM superiores a $0,062 \mathrm{ug} / \mathrm{ml}(\mathrm{P}<0,001)$. Enquanto 
que as taxas eficácia estimadas para $S$. intermedius foram de $100 \%$; $99 \%$; $51 \%$; e $0 \%$ e segundo as CIM das cepas 0,062; 0,125; 0,25 e 0,5 respectivamente. A probabilidade de obter a cura bacteriologia após o tratamento diminuiu significativamente para infeções causadas por bactérias com CIM superiores a $0.125 \mathrm{ug} / \mathrm{ml}(\mathrm{P}<$ 0,001) (Figura 3).

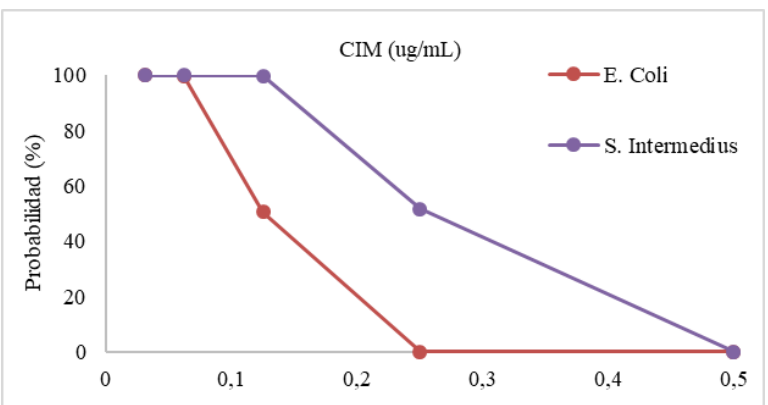

Figura 3. Probabilidade de atingir o índice $\mathrm{ASC}_{24} / \mathrm{CIM}_{90}$ após o tratamento com $2.5 \mathrm{mg} / \mathrm{kg}$ de Orbifloxacina via oral em cães segundo a CIM do microrganismo infectante baseado na simulação de Monte Carlo $(\mathrm{n}=10.000)$.

Os resultados demostraram que a doses de $5 \mathrm{mg} / \mathrm{kg}$ de difloxacina e doses de $2,5 \mathrm{mg} / \mathrm{kg}$ de orbifloxacina via oral apresentam taxas de eficácia adequadas para o tratamento de infeções por cepas de E. coli com CIM de até $0.062 \mathrm{ug} / \mathrm{ml}$ e por cepas de $S$. intermedius com CIM de até $0.125 \mathrm{ug} / \mathrm{ml}$. O analises comparativo das taxas de eficácia estimadas para os dois fármacos avaliados determinou que não existem diferencias significativas para o tratamento de infeções causadas por E. coli ou S.intermedius no intervalo de CIM estudados $(\mathrm{P}>0.05)$ (Figura 4 e 5). Estes resultados indicam que apesar da diferencia na dose administrada a eficácia de ambos tratamentos não apresenta diferencias significativas. Porem para casos com CIM superiores deveram ser realizados ajustes nas doses.

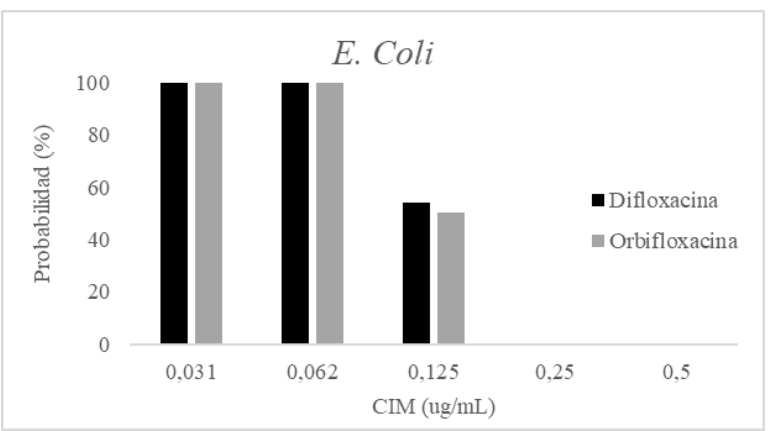

Figura 4: Probabilidade de atingir o índice $\mathrm{ASC}_{24} / \mathrm{CIM}_{90}$ após o tratamento com $5 \mathrm{mg} / \mathrm{kg}$ de difloxacina ou com 2.5 $\mathrm{mg} / \mathrm{kg}$ de orbifloxacina via oral em cães segundo a CIM da cepa de E. Coli infectante baseado na simulação de Monte Carlo $(\mathrm{n}=10.000)$.

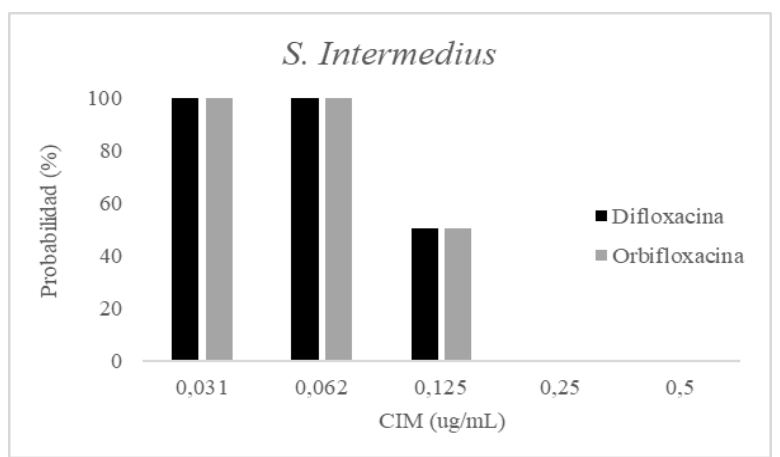

Figura 5: Probabilidade de atingir o índice $\mathrm{ASC}_{24} / \mathrm{CIM}_{90}$ após o tratamento com $5 \mathrm{mg} / \mathrm{kg}$ de difloxacina ou com 2.5 $\mathrm{mg} / \mathrm{kg}$ de orbifloxacina via oral em cães segundo a CIM da cepa de $S$. Intermedius infectante baseado na simulação de Monte Carlo $(\mathrm{n}=10.000)$.

A falha terapêutica pode ser de origem farmacocinético (PK), farmacodinâmica (PD) ou por uma combinação de ambos (Toutain et al., 2016). Mediante o analises PK/PD e segundo o índice $\mathrm{AUC}_{24} / \mathrm{CIM}_{90}$ atingido é possível identificar os casos onde a doses utilizada apresenta risco de falha terapêutica (Ahmad et al., 2016), seguidamente o ajuste de doses permitiria atingir o sucesso terapêutico assim como também a desenvolvimento de resistência (Abdelraouf et al., 2017; Balaje et al., 2013; Linder et al., 2016).

Indivíduos que apresentem alterações fisiológicas como por exemplo aumentos do debito cardíaco provocado por estados hiperdinâmicos determinam diminuição das disposição plasmática de antibióticos (Roberts et al., 2014). Por tanto, nestes casos o uso das doses propostas no presente estudo deve ser avaliado cuidadosamente principalmente para o tratamento de cepas de $S$. intermedius com CIIM de $0.125 \mathrm{ug} / \mathrm{ml}$ e de $E$. coli com CIM de $0,062 \mathrm{ug} / \mathrm{ml}$.

\section{Conclusão}

$\mathrm{O}$ presente estudo permite afirmar que apesar da diferencia na dose administrada entre difloxacina e orbifloxacina a eficácia de ambos tratamentos é equivalente. Porem para casos com CIM superiores deveram ser realizados ajustes nas doses. Isso deixa em evidencia a necessidade de incorporar no protocolo terapêutico o isolamento bacteriológico, a determinação da CIM para avaliar a necessidade otimização de doses fim 
de evitar falhas terapêuticas consequentemente potencializar desenvolvimento de resistência.

\section{Referências}

Abdelraouf, K., Linder, K. E., Nailor, M. D., \& Nicolau, D. P. 2017. Predicting and preventing antimicrobial resistance utilizing pharmacodynamics: part II Gram-negative bacteria. Expert Opinion on Drug Metabolism \& Toxicology, 13(7), 705-714.

Ahmad, I., Huang, L., Hao, H., Sanders, P., \& Yuan, Z. (2016). Application of PK/PD modeling in veterinary field: dose optimization and drug resistance prediction. BioMed Research International, 2016, 1-12.

Balaje, R. M., Sidhu, P. K., Kaur, G., \& Rampal, S. 2013. Mutant prevention concentration and PKPD relationships of enrofloxacin for Pasteurella multocida in buffalo calves. Research in Veterinary Science, 95(3), 1114-1124.

Dudley, M. N., \& Ambrose, P. G. 2000. Pharmacodynamics in the study of drug resistance and establishing in vitro susceptibility breakpoints: ready for prime time. Current Opinion in Microbiology, 3(5), 515-521.

Evans, T., \& Chapple, N. 2002. The animal health market. Nature Reviews Drug Discovery, 1(12), 937-938.

Frazier, D. L., Thompson, L., Trettien, A., \& Evans, E. L. 2000. Comparison of fluoroquinolone pharmacokinetic parameters after treatment with marbofloxacin, enrofloxacin, and difloxacin in dogs. Journal of Veterinary Pharmacology and therapeutics, 23(5), 293-302.

Gieschke, R., \& Serafin, D. 2014. Pharmacologic modeling. In Ronald, G. \& Daniel S. (Eds.), Development of Innovative Drugs via Modeling with MATLAB (pp. 87-141): Springer.

Heinen, E. 1999. Comparative pharmacokinetics of enrofloxacin and difloxacin as well as their main metabolites in dogs. Compendium on Continuing Education for the Practicing Veterinarian, 21(12), 12-18.

Heinen, E. 2002. Comparative serum pharmacokinetics of the fluoroquinolones enrofloxacin, difloxacin, marbofloxacin, and orbifloxacin in dogs after single oral administration. Journal of Veterinary Pharmacology and Therapeutics, 25(1), 1-5.
Linder, K. E., Nicolau, D. P., \& Nailor, M. D. 2016. Predicting and preventing antimicrobial resistance utilizing pharmacodynamics: Part I gram positive bacteria. Expert Opinion on Drug Metabolism \& Toxicology, 12(3), 267-280.

Martinez, M., McDermott, P., \& Walker, R. 2006. Pharmacology of the fluoroquinolones: a perspective for the use in domestic animals. The Veterinary Journal, 172(1), 10-28.

Roberts, J. A., Abdul-Aziz, M. H., Lipman, J., Mouton, J. W., Vinks, A. A., Felton, T. W., . . . Schentag, J. J. 2014. Individualised antibiotic dosing for patients who are critically ill: challenges and potential solutions. The Lancet Infectious Diseases, 14(6), 498-509.

Scholar, E. M. 2002. Fluoroquinolines: past, present and future of a novel group of antibacterial agents. American Journal of Pharmaceutical Education, 66(2), 164-171.

Silva, J., \& Hollenbach, C. 2010. Fluoroquinolonas resistência bacteriana na medicina veterinária. Arquivo do Instituto Biológico, São Paulo, 77(2), 363-369.

Toutain, P.-L., Bousquet-Mélou, A., \& Martinez, M. 2007. AUC/MIC: a PK/PD index for antibiotics with a time dimension or simply a dimensionless scoring factor? Journal of Antimicrobial Chemotherapy, 60(6), 1185-1188.

Toutain, P.-L., Ferran, A. A., Bousquet-Melou, A., Pelligand, L., \& Lees, P. 2016. Veterinary medicine needs new green antimicrobial drugs. Frontiers in Microbiology, 7, 1-16.

Toutain, P. L., Potter, T., Pelligand, L., Lacroix, M., Illambas, J., \& Lees, P. 2017. Standard PK/PD concepts can be applied to determine a dosage regimen for a macrolide: the case of tulathromycin in the calf. Journal of Veterinary Pharmacology and Therapeutics, 40(1), 16-27.

Trang, M., Dudley, M. N., \& Bhavnani, S. M. 2017. Use of Monte Carlo simulation and considerations for PK-PD targets to support antibacterial dose selection. Current Opinion in Pharmacology, 36, 107-113.

Trouchon, T., \& Lefebvre, S. 2016. A review of enrofloxacin for veterinary use. Open Journal of Veterinary Medicine, 6(2), 40-58.

Recebido: 23 Setembro, 2018

Aprovado: 25 Outubro, 2018.

Publicado: 10 Dezembro, 2018.

Licenciamento: Este artigo é publicado na modalidade Acesso Aberto sob a licença Creative Commons Atribuição 4.0 (CC-BY 4.0), a qual permite uso irrestrito, distribuição, reprodução em qualquer meio, desde que o autor e a fonte sejam devidamente creditados. 